CENTRO

HOSPITALAR

VILA NOVA DE GAIA|ESPINHO

\title{
Non-invasive cerebral oxygenation might reflect global perfusion and hemodynamic conditions
}

\author{
Costa D., Boa A., Coimbra L.
}

Centro Hospitalar de Vila Nova de Gaia/Espinho EPE

Anaesthesiology Department

BACKGROUND: Transcatheter aortic valve implantation (TAVI) emerged as minimally invasive treatment for aortic stenosis in high surgical risk patients. Near-infrared spectroscopy (NIRS) is used for noninvasive monitoring of cerebral oxygenation and may reflect global perfusion and hemodynamic conditions in critical patients. $^{1}$

IDENTIFICATION: 68 years old female; ASA IV; medical past of valvular aortic stenosis, poor ventricular function and renal chronic disease

\section{CASE REPORT:}

* Admitted at intensive care with cardiogenic shock

* Transesophageal echocardiography (TE) confirmed progression of the aorta stenosis (LV/AO gradient 79/49), LVEF 23\% and thrombus on left ventricle

* Aortic valve replacement was refused due to EUROSCORE II

* Patient was submitted to life-saving percutaneous aortic balloon valvuloplasty, despite contraindications

* Owing to the improvement on hemodynamics and echocardiographic (LV/AO gradient 40/13) it was decided to proceed urgently to TAVI with a filter in aortic arch and TE

* Intervention was performed under general anesthesia with traqueal intubation. Monitoring was held with ASA standard, invasive arterial

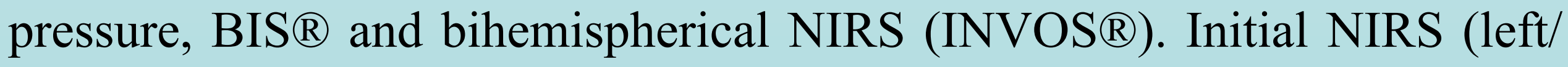
right hemisphere) was $15 / 14$, blood pressure $112 / 72 \mathrm{mmHg}$ and saturation $95 \%$. In order to achieve the endpoint of $60 \%$ on cerebral oximetry, regardless hemodynamic and respiratory values, vasopressor/inotropic was started and adjustments on ventilator parameters were made

* During the implantation of valve Edwards Sapiens 3® 26mm, hemodynamic stability was reported with surprisingly good recover after rapid ventricular pacing and a remarkable enhancement of more than $250 \%$ of the baseline of NIRS

* Discharge was 11 days after, patient was on class I/IV NYHA without major neurologic deficits

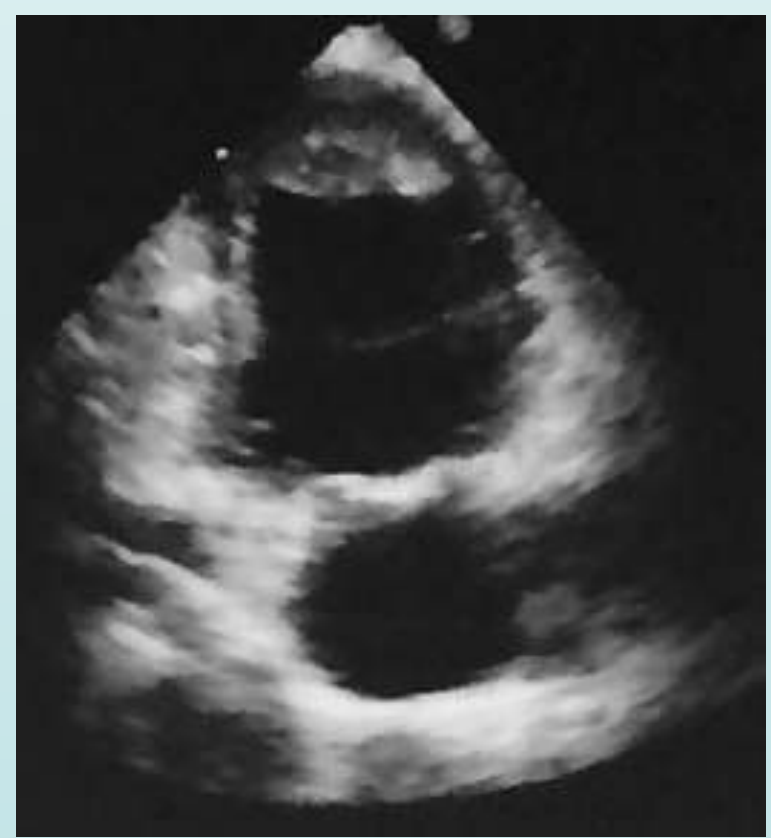

Fig. I - Thrombus on left ventricle

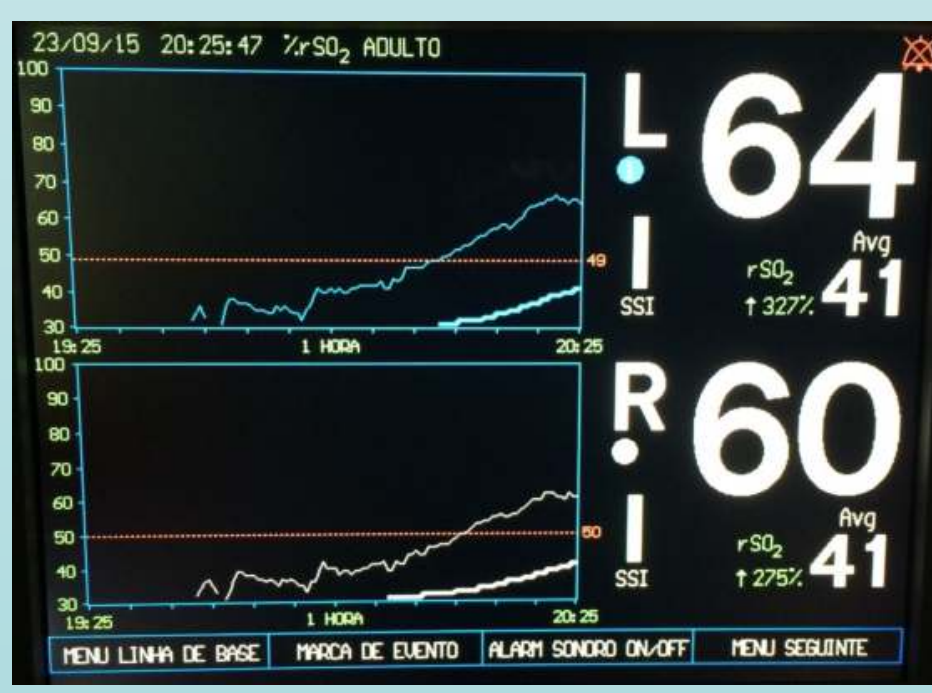

Fig. 2 - Enhancement of more than $250 \%$ of the baseline of NIRS

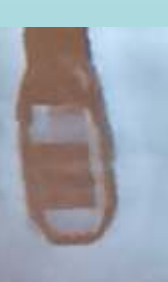

